\title{
Dynamic intelligence towards merging cloud and communication services
}

\author{
Ching-Hsien Hsu • Jianhua Ma • Mohammad S. Obaidat
}

Published online: 21 February 2014

(C) Springer Science+Business Media New York 2014

\section{Introduction}

The ever-growing next generation Cloud and Communication Services (CCS) provide dynamic intelligence and play an increasingly critical role in all aspects of our lives. Following ubiquitous computing, sensors, e-tags, networking, big data, wireless communications and cloud services is a road towards a smart world created on both cyberspaces and real spaces.

"Cloud" is a common metaphor for an Internet accessible infrastructure (e.g. data storage and computing hardware), which is hidden from users. Cloud computing makes data truly mobile and a user can simply access a chosen cloud with any Internet accessible device. In Cloud Computing, IT-related capabilities are provided as services, accessible without requiring detailed knowledge of the underlying technology. Thus, many mature technologies are used as components in Cloud computing, but still there are many unresolved and open problems.

Cloud service is a new cross-discipline that covers the science and technology needed to bridge the gap between business services and IT/telecommunication services. The underneath breaking technology suite includes web services and service-oriented architecture (SOA), cloud computing, business consulting methodology and utilities, business

\author{
C.-H. Hsu $(\bowtie)$ \\ Department of Computer Science and Information Engineering, \\ Chung Hua University, Hsinchu City, Taiwan \\ e-mail: robertchh@gmail.com \\ J. Ma \\ Department of Computer and Information Science, Hosei University, \\ Tokyo, Japan \\ e-mail: jianhua@hosei.ac.jp \\ M. S. Obaidat \\ Department of Computer Science and Software Engineering, \\ Monmouth University, West Long Branch, NJ, USA \\ e-mail: obaidat@monmouth.edu
}

process modeling, transformation and integration. The goal of cloud and services computing is to develop new computing technology and thereby enable more advanced IT/ telecommunication services to support business services more efficiently and effectively.

Today's IT paradigm, computation and communication are embedded in the environment such that all objects can be connected and always online. This will greatly facilitate intelligent monitoring and control of different objects. On the other hand, the vision of all objects connected offers not only the potential of enable emergent applications that dynamically adapts to the environments and the contexts of their usage, but also poses a significant challenge to the collection, storage and processing of enormous amounts of data. By taking advantage of virtualized resources, cloud computing services presents an attractive means to address the challenges while realizing the potential of cloud and communication services. The CCS paradigm can be generalized to include mobile devices, which not only incorporate sophisticated methods for users to interact with the online world through numerous applications in their devices, but also are endowed with multiple sensors that enable them to contribute data as nodes in the CCS. In this context, mobile cloud services that enable widespread data collection through mobile devices, and collaborative use of mobile devices to enhance existing and realize new applications are very much of interest. It is anticipated that such mobile cloud services will enrich social networking applications, encourage the widespread adoption of crowdsourcing and crowd sensing, and facilitate ubiquitous access to multimedia entertainment contents. Integration of CCS and mobile services on the cloud is expected to efficiently enable e-health, intelligent transportation, and smart power grids, which form the basis of the future smart society.

Cloud and communication services are paving the way towards a smart world in which computational intelligence is distributed throughout the physical environment to provide 
reliable and relevant services to people. This dynamic intelligence will change the computing landscape because it will enable new breeds of applications and systems to be developed and the realm of computing possibilities will be significantly extended. By enhancing everyday objects with intelligence, many tasks and processes could be simplified, the physical spaces where people interact like the workplaces and homes, could become more efficient, safer and more enjoyable.

Despite a wide body of research development effort, ensuring the communications of cyberspaces and real spaces, how to merge cloud and communication services remains an open challenge. This special issue is in response to the increasing convergence between cloud and communication services, while different approaches exist, challenges and opportunities are numerous in this context. The research papers selected for this special issue represent recent progress in the field, including works on cloud architectures, mobile computing, security issues, services computing and modeling, resources management, P2P technologies and applications, among others. All of these papers not only provide novel ideas and state-of-the-art techniques in the field, but also stimulate future research in merging cloud and communication services.

\section{Cloud architecture}

Service clouds are usually distributed infrastructure, which deploy communication services in clouds. The scalability is an important characteristic of service clouds. With the scalability, the service cloud can offer on demand computing power and storage capacities to different services. The paper by Yang et al. (2014) entitled "A Cost-Aware Auto-Scaling Approach using the Workload Prediction in Service Clouds" presented a novel service cloud architecture and a linear regression model for predicting the workload. Based on the predicted workload, an auto-scaling mechanism is proposed to scale virtual resources at different resource levels in service clouds. The auto-scaling mechanism combines the real-time scaling and the pre-scaling. Experimental results are provided to demonstrate that the proposed approach can satisfy users Service Level Agreement (SLA) while keeping scaling costs low.

Byzantine consensus is a well-known problem in distributed systems. An important factor that may influence the performance of Byzantine fault tolerance algorithms is scalability. The paper by Lim et al. (2014) entitled "Scalable and Leaderless Byzantine Consensus in Cloud Computing Environments" presents a scalable and leaderless Byzantine consensus implementation. Unlike conventional distributed systems, the network topology of cloud computing systems is often not fully connected, but loosely coupled and layered. The message complexity of the proposed Byzantine consensus scheme is relative low. Experimental results and correctness proof show that the proposed scheme can solve the Byzantine consensus problem safely in a scalable way without a bottleneck and a leader in cloud computing environments.

Data centres have become more and more popular for storing large volumes of data. Companies like Google, Microsoft, Yahoo, and Amazon use data centres for web search, storage, ecommerce, and large-scale general computations. Building data centres using commodity TCP/IP and Ethernet networks is still attractive because of their low cost and ease of use. However, TCP is easily to suffer a drastic reduction in throughput when multiple senders communicate with a single receiver in these networks, which is termed incast. The paper by Wang et al. (2014a, b) entitled "IDTCP: An Effective Approach to mitigating the TCP Incast Problem in Data Center Networks" analyses the TCP incast problem in data centers by focusing on the relationship between the TCP throughput and the congestion control window size of TCP. The rationality of the analysis is verified by simulations which provide significant implications to the TCP incast problem. Based on these implications, an effective approach, termed as Incast Decrease TCP, is proposed to mitigate the TCP incast problem. Analysis and simulation results verify that the proposed approach effectively mitigates the TCP incast problem and noticeably improves the TCP throughput.

In regard to Peer-to-Peer architecture, in order to deal with the scalability problem, i.e., continuous growth of participating peers, the paper by Teng et al. (2014) entitled "A SelfSimilar Super-Peer Overlay Construction Scheme for Super Large-Scale P2P Applications" proposes a scalable hierarchical unstructured P2P system in which a self-similar square network graph is proposed to construct and maintain the super-peer overlay topology adaptively. The proposed topology is a constant-degree topology in which each node maintains a constant number of neighbor nodes. In addition, a simple and efficient message-forwarding algorithm is presented to ensure each super-peer to receive just one flooding message. The analytical results showed that the proposed SSNG-based overlay is more scalable and efficient than the perfect difference graph-based overlay proposed in the literature.

\section{Mobile cloud and services}

Mobile applications and services relying on mobility prediction have recently spurred lots of interest. Predicting mobile phone users' locations in next few hours is essential for a wide range of mobile applications, including location-based service, mobile access control, mobile multimedia QoS provision, as well as resource management for mobile computation and storage. The paper by Xiong et al. (2014) entitled "MPaaS: Mobility Prediction as a Service in Telecom Cloud" proposes mobility prediction based on cellular traces as an infrastructural level service of telecom cloud. The proposed Mobility Prediction as a Service (MPaaS) embeds mobility mining and forecasting algorithms into a cloud-based user 
location-tracking framework. The authors take Mobility aware Personalization and Predictive Resource Allocation as key features to elaborate on how MPaaS drives new fashion of mobile cloud applications. The preliminary study has observed that the MPaaS system can enhance telecom cloud with large-scale mobility prediction capacity.

On the other hand, the proliferation of ubiquitous Internet access has enabled millions of Web users to perform various activities of daily life, where many of these activities result in the construction of large repositories of knowledge. Thus, this knowledge represents a valuable source of information. Today, most of the current retrieval approaches are targeted only to Web services, leaving out different other resource types. The paper by Suarez-Meza et al. (2014) entitled "Fully Automated Resource Retrieval in Telecommunications and Internet Converged Environments" proposes an innovative approach for resource retrieval over Telecommunications and Internet converged environments, which brings together traditional NLP techniques with retrieval process based on lightweight semantic approaches. The preliminary experiments show promising results in contrast to traditional approaches.

Today, smart phones have become increasingly popular in our daily life. They offer users more powerful processors, larger memory, multi-network interfaces and a wide variety of operating systems. With prosperity of applications on smart- phones, energy saving for smart phones has drawn in- creasing attention. The paper by Xia et al. (2014) entitled "Phone2Cloud: Exploiting Computation Offloading for Energy Saving on Smartphones in Mobile Cloud Computing" introduces Phone2Cloud, a computation offloading-based system for energy saving on smart phones in the context of mobile cloud computing. The main idea of Phone2Cloud is to offload computation of an application running on smart phones to the cloud. The objective is to improve energy efficiency of smart phones and at the same time, enhance the application's performance through reducing its execution time. The authors implemented the prototype of Phone2Cloud on Android and Hadoop environment. The experimental results show that Phone2Cloud can effectively save energy for smart phones and reduce the application's execution time.

\section{Security and QoS}

There are now many applications deployed in a network environment. They need to be secure when communication takes place between users and servers. As a consequence, a strong protocol guarantees that users and service providers are secure against many kinds of attacks is of critical importance. A wellknown authentication scheme is based on dynamic identity to prevent transactions from being intercepted by malicious users. The paper by Truong et al. (2014) entitled "Enhanced Dynamic Authentication Scheme (EDAS)" proposes a novel authentication scheme to enhance security and overcome limitations of existing approaches. Security analysis shows that the proposed method can efficiently resist known types of attacks. Experimental results also show that the method can be implemented and processed in real-time thus applicable for not only regular computers, but also mobile devices.

The emergence of cloud data centres enhances the capability of online data storage. Since massive data is stored in datacentres, it is necessary to effectively locate and access interest data in such a distributed system. However, traditional search techniques only allow users to search images over exactmatch keywords through a centralized index. The paper by Liao et al. (2014) entitled "A Scalable Approach for Content Based Image Retrieval in Cloud Datacenter" proposes a scalable image retrieval framework which can efficiently support content similarity search and semantic search in the distributed environment. Its key idea is to integrate image feature vectors into distributed hash tables (DHTs) by exploiting the property of locality sensitive hashing (LSH). Thus, images with similar content are most likely gathered into the same node without the knowledge of any global information. This paper shows that the proposed approach yields high recall rate with good load balance and only requires a few number of hops.

In addition to security issues, QoS are considered as key issue on building service-oriented architecture systems. The rising development of service-oriented architecture makes more and more alternative Web services offered with equivalent or similar functions. Therefore, how to select a feasible service to meet the demands of different users has become a popular research area. The paper by Wang et al. (2014a, b) entitled "Web Service Selection Based on Multi-QoS Prediction" proposes a new framework for multi-user Web services selection. This framework predicts missing multi-QoS values according to the historical QoS experience from different users, and then selects the global optimal solution for multi-user by the proposed fast match approach. Comprehensive empirical studies demonstrate the utility of the proposed method.

\section{Conclusions}

All of the above papers address either original research in cloud or communication services or propose novel application models in the various mobile, ubiquitous and security fields. They also trigger further related research and technology improvements in application of cloud computing and communication services. This special issue serves as a landmark source for education, information, and reference to professors, researchers and graduate students interested in updating their knowledge about or active in cloud/services computing, resource provisioning and management, and novel application models for merging cloud and communication services and systems.

This special issue of Information Systems Frontiers covers different aspects of the problem; both from the 
theoretical and practical side. After a large open call we received 54 submissions, ten research papers were selected by an international editorial review committee. Each paper was reviewed by at least three reviewers. An acceptance rate of $18.5 \%$ has resulted from the selection.

The guest editors would like to express sincere gratitude to Dr. Ram Ramesh (EiC, ISF), for giving the opportunity to prepare this special issue. In addition, we are deeply indebted to numerous reviewers for their professional effort, insight and hard work to put together these selected articles. Last but not least, we are grateful to all authors for their contributions and for undertaking two-cycle revisions of their manuscripts, without which this special issue could not have been produced. We hope that this special issue will be a good addition to the area of next generation Cloud and Communication Services.

\section{References}

Liao, J., Yang, D., Li, T., Wang, J., Qi, Q., \& Zhu, X. (2014). A scalable approach for content based image retrieval in cloud datacenter. Information Systems Frontiers, 16(1). doi:10. 1007/s10796-013-9467-0.

Lim, J., Suh, T., Gil, J., \& Yu, H. (2014). Scalable and leaderless Byzantine consensus in cloud computing environments. Information Systems Frontiers, 16(1). doi:10.1007/s10796-013-9460-7.

Suarez-Meza, L. J., Zúñiga, J. A., Pedraza, E. C., \& Corrales, J. C. (2014). Fully automated resource retrieval in telecommunications and internet converged environments. Information Systems Frontiers, 16(1). doi:10.1007/s10796-013-9457-2.

Teng, H.-Y., Lin, C.-N., \& Hwang, R.-H. (2014). A self-similar superpeer overlay construction scheme for super large-scale P2P applications. Information Systems Frontiers, 16(1). doi:10.1007/s10796013-9456-3.

Truong, T.-T., Tran, M.-T., \& Duong, A.-D. (2014). Enhanced dynamic authentication scheme (EDAS). Information Systems Frontiers, 16(1). doi:10.1007/s10796-013-9461-6.

Wang, S., Hsu, C.-H., Liang, Z., Sun, Q., \& Yang, F. (2014). Multi-user web service selection based on multi-QoS prediction. Information Systems Frontiers, 16(1). doi:10.1007/s10796-013-9455-4.

Wang, G., Ren, Y., Dou, K., \& Li, J. (2014). IDTCP: an effective approach to mitigating the TCP incast problem in data center networks. Information Systems Frontiers, 16(1). doi:10.1007/s10796-013-9463-4.

Xia, F., Ding, F., Li, J., Kong, X., Yang, L. T., \& Ma, J. (2014). Phone2Cloud: exploiting computation offloading for energy saving on smartphones in mobile cloud computing. Information Systems Frontiers, 16(1). doi:10.1007/s10796-013-9458-1.

Xiong, H., Zhang, D., Zhang, D., Gauthier, V., Yang, K., \& Becker, M. (2014). MPaaS: mobility prediction as a service in telecom cloud. Information Systems Frontiers, 16(1). doi:10.1007/s10796-0139476-z.

Yang, J., Liu, C., Shang, Y., Cheng, B., Mao, Z., Liu, et al. (2014). A costaware auto-scaling approach using the workload prediction in service clouds. Information Systems Frontiers, 16(1). doi:10.1007/ s10796-013-9459-0.

\section{Editorial review board}

Haider Abbas

Saeid Abolfazli

Javier Alonso

Ashish Bagwari

Vaibhav Bhatnagar

Ing-Chau Chang

Chin-Ling Chen

Jiann-Liang Chen

Der-Jiunn Deng

Yuhui Deng

Jie Ding

Salvatore Distefano

Yong-Yi FanJiang

$\mathrm{Xin} \mathrm{Gu}$

Nien-Lin Hsueh

John Huh

Chung-Ming Hwang

Sadeeq Jan

YoungSik Jeong

Nesrine Khabou

Chin-feng Lai

Cheng-Chi Lee

Chun-Ta Li

Wang Li

Yun Li

Xiaofei Liao

JongBeom Lim

Jyh-Shyan Lin

Shu Bo Liu

Yang Liu

Jung-Wen Lo

Jian $\mathrm{Lu}$

Shang-Pin Ma

Zexiang Mao

Rahat Massed

Saravanan Muthaiyah

Lei Ning

Yong Peng

Pit Pichappan

Qasim Rajpoot

Raihan Rasool

Mohamed Sellami

Muhammad Awais Shibli

Lei Tian

Promod Tiwari

Toan Thinh Truong

Salvatore Venticinque

Cho-Li Wang

Guodong Wang

Leandro Krug Wives

Peter Charles Woods

Feng Xia

Haoyi Xiong

Di Yang

Fuw-Yi Yang

Jingqi Yang

Morcous M. Yassa

Qinglong Zhan

Mohamed Faten Zhani

Degan Zhang

Xiao-yong Zhang

Mohamed Faten Zhani

Zhangbing Zhou

Yi Zhu 
Professor Ching-Hsien (Robert) Hsu is a professor in department of computer science and information engineering at Chung Hua University, Taiwan; and distinguished chair professor in school of computer and communication engineering at Tianjin University of Technology, China. His research includes high performance computing; cloud computing, parallel and distributed systems, ubiquitous/pervasive computing and intelligence. He has published 200 papers in refereed journals, conference proceedings and book chapters in these areas. He has been involved in more than 100 conferences and workshops as various chairs and more than 200 conferences/workshops as a program committee member. He is the editor-in-chief of international journal of Grid and High Performance Computing, and international journal of Big Data Intelligence; and serving as editorial board for around 20 international journals. He has been acting as an author/co-author or an editor/co-editor of ten books from Springer, IGI Global, World Scientific and McGraw-Hill. He has also edited a number of special issues at top journals, such as IEEE Transactions on Cloud Computing, IEEE Transactions on Services Computing, Future Generation Computer Systems, Journal of Supercomputing, International Journal of Communication Systems, Automated Software Engineering, Journal of System Architecture, Concurrency and Computation: Practice and Experience, The Knowledge Engineering Review, Internet Research, Information System Frontiers, etc. He was awarded five times annual outstanding research award through 2005 to 2012 and a distinguished award in 2008 for excellence in research from Chung Hua University. He has been serving as executive committee of Taiwan Association of Cloud Computing (TACC) from 2008 to 2012; executive committee of the IEEE Technical Committee of Scalable Computing (2008-2012). He is member of Phi Tau Phi Scholastic honor society; IEEE senior member; regional director of the Future Technology Research Association (FTRA); and standing director of Taiwan Association of Cloud Computing (TACC).

Prof. Jianhua Ma received his B.S. and M.S. degrees of Communication Systems from National University of Defense Technology (NUDT), China, in 1982 and 1985, respectively, and the $\mathrm{PhD}$ degree of Information Engineering from Xidian University, China, in 1990. He has joined Hosei University since 2000, and is currently a professor at Digital Media Department in the Faculty of Computer and Information Sciences. Prior to joining Hosei University, he had 15 years' teaching and/or research experiences at NUDT, Xidian University, and The University of Aizu, Japan. He is teaching the courses of Java Programming, Computer Networks, Multimedia Technologies and Applications, Ubiquitous Computing, Advanced Networking and Computing, Project A/B, and Research Seminars of Advanced Web/Cyber and Ubiquitous Computing. $\mathrm{He}$ is a supervisor of doctor and master students. Dr. Ma is a member of IEEE and ACM. He has edited over 15 books/proceedings, and published more than 200 academic papers in journals, books and conference proceedings. He has delivered about 20 keynote speeches in international conferences, and given invited talks in over 40 universities/institutes. He was awarded as the Excellent Graduated Student by NUDT in 1982 . He received the Annual Excellent Paper Awards from China Information Theory Society, Electronics Society, and Association of Hunan Science and Technology in 1985, 1986 and 1991, respectively. He received the Best Paper Award from the 2000 International Conference on Information Society in the 21st Century: Emerging Technologies and New Challenges, and the Highly Commended Paper Award from the 2004 I.E. International Conference on Advanced Information Networking and Applications. He is the Chair of IEEE Task Force on Autonomic and Trusted Computing, a co-founder of IEEE CIS Task Force on Ubiquitous Intelligence and Computing, and received the Certificate of Appreciation from IEEE Computer Society in 2004-2007.

Professor Mohammad S. Obaidat (Fellow of IEEE and Fellow of SCS) is an internationally well-known academic/researcher/ scientist. He received his Ph.D. and M. S. degrees in Computer Engineering with a minor in Computer Science from Ohio State University. He has received extensive research funding and has published about twenty five (25) books and about six hundreds (600) refereed technical articles. Mohammad is the Editor-in-Chief of three scholarly journals and is also an editor, advisory editor of numerous international journals and transactions including IEEE journals/transactions such as IEEE Wireless Communications and IEEE Systems Journal. He has chaired numerous international conferences and given numerous keynote speeches all over the world. Between 2009 and 2011, he served as the President of the Society for Modeling and Simulation International, SCS. He is an ACM and SCS Distinguished Lecturer/speaker and served as an IEEE Distinguished Speaker. He served as ABET/CSAB Program Evaluator. He has received worldwide numerous awards for his technical contributions including Nokia Research Fellowship and the distinguished Fulbright Scholar Award. He received the SCS Outstanding Service Award for his excellent leadership, services and technical contributions to modeling and simulation of computer systems and networks. Dr. Obaidat received in 2009 the Society for Modeling and Simulation Intentional (SCS) prestigious McLeod Founder's Award in recognition of his outstanding technical and professional contributions to modeling and simulation. He received in Dec 2010, the IEEE ComSoc- GLOBECOM 2010 Outstanding Leadership Award for his outstanding leadership of Communication Software Services and Multimedia Applications Symposium, CSSMA 2010. Four of his recent papers have received the best paper awards in the IEEE GLOBCOM 2009, IEEE AICCSA 2009, IEEE DCNET 2011 and IEEE CITS 2013 international conferences. He received very recently (2012) the SCS's prestigious Presidential Service Award for his outstanding unique, long-term technical contributions and services to the profession and society. 